\title{
Nurse-led continuum of care for people with diabetes and pre-diabetes in Nepal (NUCOD): study protocol for a cluster randomized controlled trial
}

\section{Dong (Roman) Xu}

Sun Yat-Sen University

Rubee Dev

Sun Yat-Sen University

Abha Shrestha

Kathmandu University School of Medical Sciences

\section{Lingling Zhang}

University of Massachusetts Boston

\section{Archana Shrestha}

Harvard University T H Chan School of Public Health

Pushpanjali Shakya

Kathmandu University School of Medical Sciences

James Hughes

University of Washington

\section{Prabin Shakya}

Seoul National University College of Medicine

Jinghua Li

Sun Yat-Sen University

\section{Jing Liao}

Sun Yat-Sen University

Biraj Karmacharya ( $\nabla$ birajmk@kusms.edu.np )

Kathmandu University

\section{Study protocol}

Keywords: Continuum of care; Diabetes, Pre-diabetes, Randomized controlled trial, Implementation

Posted Date: May 8th, 2020

DOI: https://doi.org/10.21203/rs.2.21326/v2

License: (1) (1) This work is licensed under a Creative Commons Attribution 4.0 International License. Read Full License 
Version of Record: A version of this preprint was published at Trials on May 29th, 2020. See the published version at https://doi.org/10.1186/s13063-020-04372-5. 


\section{Abstract}

Background: The purpose of this study will be to improve diabetes prevention, access to care and advocacy through a novel cost-effective nurse-led continuum of care approach that incorporates diabetes prevention, awareness, screening and management for low-income settings, and furthermore utilizes the endeavor to advocate for establishing standard diabetes program in Nepal.

Methods: We will conduct a two-arm, parallel group, stratified cluster randomized controlled trial of a NUrse-led

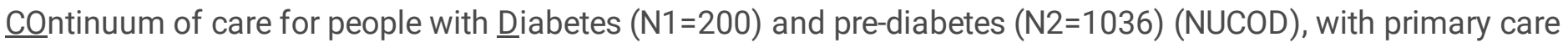
centers ( 9 Outreach Centers and 17 Government Health Posts) as a unit of randomization. NUCOD will be delivered through the trained diabetes nurses in the community to the intervention group and the outcomes will be compared to the usual treatment group at 6 and 12 months of the intervention. The primary outcome will be change in glycated hemoglobin (HbA1c) level among diabetes and progression to type 2 diabetes among prediabetes, and implementation outcomes measured using the RE-AIM (reach, effectiveness, adoption, implementation, and maintenance) framework. Outcomes will be analyzed on an intention to treat basis.

Discussion: The results of this trial will provide information about the effectiveness of the NUCOD program in improving clinical outcomes for diabetes and prediabetes individuals, and implementation outcomes for the organization. The continuum of care model can be used for the prevention and management of diabetes and other non-communicable diseases within and beyond Nepal with similar context.

Trial registration: This study was registered in a clinical trials registry via ClinicalTrials.gov (NCT04131257) on 18 October 2019. URL: https://bit.ly/34qwIAd

\section{Background}

Prevalence of type 2 diabetes (hereafter, diabetes) has been rising like a silent epidemic in Nepal similar to other developing countries. A systematic review and meta-analysis reported a pooled prevalence of type 2 diabetes of 8.4\% (95\% Cl: 6.2-10.5\%) [1]. It is estimated that between 1990 to 2010 the burden of diabetes has increased by about $89 \%$ in Nepal [2].

The recently released Disease Control Priority-3 (DCP-3) systematically reviewed available interventions for diabetes in resource-limited settings and have recommended strongly for the following interventions based on their cost-effectiveness and feasibility: (i) targeted (two-steps) screening for both prediabetes and diabetes; (ii) blood pressure control among people with diabetes; (iii) lifestyle interventions to prevent diabetes among high-risk individuals; (iv) good glycemic control along with smoking cessation and foot care. Those interventions, ranging from case detection to prevention of diabetes among those at high risk to diabetes management and to screening for preventing diabetes complications, have been extensively tested for efficacy and cost-effectiveness. However, DCP-3 pointed out two knowledge gaps: little evidence for the testing of the effectiveness of those interventions in low-income settings, and lack of implementation-oriented research for adopting and scaling up those interventions. This is compounded by the fact that chronic disease like diabetes needs a structured, well-concerted comprehensive approach comprising of the continuum of care prevention activities, timely screening, standard clinical management, and self-management skills for lifestyle modification rather than segregated interventions. Nurse care coordinators have been found to improve patient and health service outcomes, particularly when they frequently interacted with the patients, conducted follow-up with monitoring and education of the disease and 
behavioral changes, and involved transition care [3-7]. However, they often focused on specific components rather than the full spectrum of the continuum of care for the patient. Also, studies are rarely conducted in low-income countries.

Dhulikhel Hospital (DH) system is a community-oriented, non-governmental health system with an extensive network of 21 rural community-based outreach centers. The DH system provides a well-suited setting for innovating continuum of care for people with diabetes. In this trial, we aim to develop and implement a "continuum of care" program with trained nurses as the nucleus. The nurses, along with the community health worker, will coordinate and implement a composite of DCP-3 recommended individual intervention components for the lowincome setting: (i) leading community awareness campaigns; (ii) organizing diabetes screening events; (iii) linking diabetic patients to comprehensive clinical care (which will have been structured based on standard guidelines); and (iv) ensuring treatment adherence and self-management through regular community-based group counselling programs. The primary objective of this study is to examine the effectiveness of this comprehensive intervention in promoting the health of the people with diabetes and prediabetes, and the secondary objective is to examine whether the nurse coordination will promote the implementation of the evidence-based, DCP-3 recommended interventions. We hypothesize that the NUrse-led COntinuum of care for people with Diabetes program (NUCOD) will improve both clinical outcomes as well as implementation outcomes, compared with a diabetes usual care in low-income settings.

\section{Methods}

\section{Design}

This will be a two-armed, parallel group, stratified cluster randomized controlled trial (RCT) of a NUCOD program (Figure 1), with health center as a unit of randomization. We will use a computer-generated list of random numbers to randomize health centers stratified by Kathmandu University (KU) outreach centers and Government health posts, whereby all patients from the same health center will be allocated to the same group. The study will be planned and implemented in concordance with the Consolidated Standards of Reporting Trials (CONSORT) cluster trial extension statement [8] and Standards for reporting implementation studies (StaRI) statement [9]. We plan to use the cluster design to reduce between-group contamination and spill-over effects of the intervention as well as to align the design with the natural project implementation unit based on the cluster.

\section{Setting}

The study will be conducted in the 26 clusters- 9 outreach centers (Bahunepati, Manekharka, Hindi Health, Baluwa, Bolde Phediche, Dapcha, Kartike Deurali, Salambu, and Dhunkharka Health Centers) of Dhulikhel Hospital, Kathmandu University Hospital and 17 Government health posts located in the adjoining catchment areas will be purposively selected from the Kavrepalanchowk and Sindhupalchowk districts. The primary stratification variable, as well as the unit (cluster) of randomization and implementation, will be health clinics (outreach centers and health posts [HP]). There will be 13 clusters per treatment arm (1:1 allocation ratio). Outreach centers are the small medical health facilities that offer basic and emergency care services in remote areas of Nepal. These centers are run by the Kathmandu University Hospital. Similarly, health posts are the Government-run facilities that provide basic health care and preventive medication in remote rural areas of Nepal. 
Kavrepalanchowk district located in Province 3 of Nepal consists of 13 municipalities; 6 urban and 7 rural municipalities with a total of 137 wards. The headquarter of this district is Dhulikhel municipality. A total of 381,937 population resides in this district; $62.51 \%$ live in urban and $37.49 \%$ live in rural municipalities. There is one 15-bed District Hospital, 4 Primary Health Care (PHC), and 86 HPs in the district [10]. Sindhupalchowk district also located in Province 3 of Nepal consists of 12 municipalities; 3 urban and 9 rural municipalities with a total of 103 wards. The headquarter of Sindhupalchowk district is Chautara. A total of 287,798 population resides in this district; $41.42 \%$ live in the urban municipality and $58.58 \%$ live in the rural municipality. There is one 15 bedded District Hospital, 3 PHC, and 75 HPs in the district [10]. The map of Kavrepalanchowk and Sindhupalchowk district with the distribution of outreach centers and health posts are shown in Figure 2 and Figure 3.

\section{Participants}

\section{Eligibility criteria for health centers/health posts}

- Outreach centers/health posts located in the Kavrepalanchowk and Sindhupalchowk districts

- Have a nurse (staff nurse or auxiliary nurse midwife [ANM])

\section{Eligibility criteria for participants}

To resemble the real-world settings as much as possible, we will apply the minimum inclusion and exclusion criteria for the participants. Anyone (i) with confirmed pre-existing type II diabetes and prediabetes at the time of our screening (i.e., pre-existing diabetes or prediabetes); or (ii) with a confirmed diagnosis of type II diabetes or prediabetes after our community screening process (i.e., newly diagnosed diabetes and prediabetes); and who (iii) is not planning to relocate outside of the current place of living in next 2 years; and (iv) is older than 18 years of age, will be eligible for project recruitment. People will be excluded if they (i) are not psychologically capable of communication; and (ii) are diagnosed as type 1 diabetes. Participants will be assessed for the risk of diabetes using the Indian Diabetes Risk Score (IDRS) [11]. Anyone identified as at-risk will undergo a random finger-prick glucose test followed by the hemoglobin A1c (HbA1c) test. The diagnosis will be confirmed by the HbA1c level of $\geq 6.5 \%$ for diabetes or between $5.7 \%-6.4 \%$ for prediabetes [12].

\section{Screening and Recruitment}

The program will be rolled out with each outreach center/health post serving as the implementation unit. The program participants will be recruited through a community-based screening effort. Information regarding the screening event will be disseminated at the time of the awareness campaign. Trained diabetes nurses will screen the general population at the outreach center/health post, and those screened positive will be referred to the $\mathrm{DH}$ diabetes clinic for further tests and for program recruitment if eligible for the study. All the screening procedures including lab investigations and anthropometric measurements will be carried out at the respective centers, and all the longitudinal data of patients will be entered in an Electronic Health Record (EHR) system for data storage and management. Each participant will be assigned a unique identification number at the time of enrolment. All data collected as part of this study will be identified with this number. Research assistants (RAs) will be responsible for obtaining the signed informed consent form from the participants. On the consent form, participants will be asked if they agree to use of their data should they choose to withdraw from the trial. Participants will also be asked for permission for the research team to share relevant data with people from the Universities taking part in the 
research or from regulatory authorities, where relevant. This trial involves collecting biological specimens for storage.

Recruitment will be primarily through conducting diabetes awareness mass campaigns in the targeted sites of the two districts to achieve adequate participant enrolment. We intend to reach 50,000 people (approximately 2000 people at each of the 9 outreach centers and 17 health posts). Interested candidates will contact study nurses who will provide them basic information about participation, including information on the screening process, time commitment and expectations associated with participation. Assuming $25 \%$ of those who are made aware of diabetes will participate in the screening (see below for the details of screening methods), we target to screen 12,500 participants. The two-step screening approach will be used for the study after assessing the risk of diabetes among the individuals by using the IDRS assessment tool. In the first step, anyone under risk will undergo random finger-prick blood glucose test using Beurer glucometers [13]. A random blood sugar level of $200 \mathrm{mg} / \mathrm{dL}$ or higher will be considered as positive for diabetes and sugar level of $140 \mathrm{mg} / \mathrm{dL}$ to $199 \mathrm{mg} / \mathrm{dL}$ will be considered as positive for prediabetes [12]. In the second step, participants screened positive for a random blood glucose level will undergo a glycosylated hemoglobin $(\mathrm{HbA} 1 \mathrm{c})$ test. The $\mathrm{HbA} 1 \mathrm{c}$ level between $5.7 \%$ to $6.4 \%$ will be considered as positive for prediabetes and $\mathrm{HbA} 1 \mathrm{c}$ level of $6.5 \%$ or above will be considered as positive for diabetes [12]. Participants with $\mathrm{HbA} 1 \mathrm{c}$ level $\geq 5.7 \%$ will be invited to participate in the study. Based on earlier quoted prevalence of $8.4 \%$ (95\% Cl: $6.2 \%-10.5 \%)$ for diabetes [1] and 13\% (95\% Cl: $11.8 \%-14.5 \%)$ for prediabetes [14], we expect to identify $1050(12500 * 0.084)$ individuals with diabetes and $1625(12500 * 0.13)$ individuals with prediabetes for this study.

\section{Randomization}

The 26 health clinics will be randomized 1:1 into the continuum of care group (intervention) and the usual care group (control), stratified by outreach center and health post. The usual care group is the one who continues managing their diabetes under the direction of their primary care providers, which is the most commonly used method for diabetes management in Nepal. The selection of the usual care group as a comparator is thus justified. A statistician otherwise not involved in the project will perform this group assignment at cluster level with simple random selection with the statistical program $\mathrm{R}$. Randomization will be done before participant recruitment. Outcome assessors will be blinded to the group assignment, but the nurses and patient participants will be aware of their group assignment. If blinding to the outcome assessor is accidentally broken, they will follow a standard protocol for re-assessment of the patients at another time. Any violations of the study protocol will be recorded and reported to the Ethics Committee.

\section{Baseline Assessment}

At baseline, trained RAs will interview the participants using a standardized electronic questionnaire using CommCare, mobile platform designed for data collection [15]. RAs will receive two weeks of training on data collection and ethical issues. The questionnaire will assess socioeconomic characteristics including age, sex, ethnicity, religion, marital status, annual income, education level, family history, and lifestyle factors including smoking, alcohol intake, and physical activity. We will use the Global Physical Activity Questionnaire [16], and calculate the metabolic equivalent of task (MET) minutes per week. A weekly MET equivalent of 600 would represent 30 minutes of brisk walking five times per week or 15 minutes of running five times per week. We will use Prime Screen questionnaire [17], a short diet assessment tool to assess the diet quality of the study participants. Body weight will be measured with minimum clothing and without shoes using an Omron Model HBF-400 scale 
and recorded to the nearest 0.1 pounds. The weighing scale will be calibrated to zero every day. Participants' heights will be measured, without shoes, while the participants stand against a wall. Height will be measured using a tape measure and recorded to the nearest $0.1 \mathrm{~cm}$.

Blood samples will be collected for HbA1c, low-density lipoprotein (LDL), high-density lipoprotein (HDL), triglycerides, and total cholesterol at each respective health center where all the laboratory procedures will be carried out. Blood samples will be collected using evacuated blood collection tubes. Participants will be asked to fast overnight (8-14 hours).

\section{Intervention: NUCOD Program Components}

The intervention will be delivered by specially trained nurses from the Dhulikhel Hospital (DH). The intervention has been developed based on our extensive literature review and the recommendations from the Disease Control Priorities-3 as well as an analysis of local contexts in consultation with various stakeholders. The intervention will include the following components, logically sequences as a continuum of care approach. Figure 4 presents the major program components. The nurses will act as the leader and coordinator for the implementation of all those program components.

- Training of nurses. By drawing from standard training manuals published from International Diabetes Federation and American Diabetes Association, the project management team will first develop a protocol and content for the training of nurses for this program. This training protocol will be tailored to the context of Nepal and will include case studies and examples relevant to Nepal. The training will focus on clinical as well as case management skills. We will train 26 nurses (one for each site) into diabetes-nurses using this training protocol.

- Community awareness campaigns: The nurses will lead the organization of a mass-campaign on diabetes in coordination with local newspapers, radio stations, youth-groups, municipality offices, district health offices, and other health facilities. The campaigns will, in particular, include a simple diabetes risk factor assessment tool to be adapted from the Type 2 Diabetes Risk Test (DRT) of the American Diabetes Association [18]. The campaign will encourage people with high risk to attend the screening program.

- Screening programs: The nurses will organize screening programs for both diabetes and pre-diabetes for the community members on specific dates and sites which will be communicated beforehand to the people in the locality. The screening will be done in coordination with the clinical biochemistry department of DH.

- Linkage to clinical Care: People screened positive for diabetes and prediabetes (pre-existing as well as newly) will be linked to the $\mathrm{DH}$ clinic by nurses where they will be recruited into the program and will receive their group assignment. People assigned to the control group will follow the usual procedure, while those assigned to the intervention group will attend a one-hour nurse-led counseling session on diabetic care. The patients will be responsible for their medical costs (lab investigations and medicine), while people experiencing financial hardship can apply for charity care (free or at a reduced price) with guidance from the nurse. The assigned nurse will be responsible for determining their financial profile by reviewing the annual household income, applicable assets, available insurance coverage, and confirmation of other sources of payment.

- Community follow-up counselling and support to the diabetic patients: The nurse will organize diabetic patients in groups of 10 and arrange bi-monthly meetings (each two-hour long) in which the patients will be facilitated using the 5 A framework (Assess, Advise, Agree, Assist, and Arrange follow-up) to adopt healthy lifestyle (dietary changes, physical activity, cessation of smoking, etc.) [19]. In addition to the lifestyle 
changes, the participants will also be facilitated to adhere to medical advice (timely follow-up, adherence to medications, etc.). The nurses will also coordinate with the diabetes clinic at the hospital to ensure that the clinical care and the community-level interventions complement each other. They will also have an electronic tablet that has all the details (laboratory results, behavioral parameters, anthropometric measures, etc.) of the participants so that the progress of the participants in these parameters can be recorded and assessed.

- Prevention programs for pre-diabetic participants: In addition to the people with diabetes, the nurses will also form groups of prediabetes participants and will arrange monthly meetings to help facilitate the adoption of healthy lifestyle using the Diabetes Prevention Program (DPP) curriculum. The DPP lifestyle intervention has been associated with significantly reducing the development of diabetes through its structured behavior changing approaches [20]. A multidisciplinary team of dieticians and physical therapists will assist with the other components of the DPP program that are related to nutrition and physical activity.

The control group will receive the diabetic usual care, which includes the same hospital quality improvement for diabetic care as mentioned above, the follow-up services from the Female Community Health Volunteers (FCHVs) (but without the nurse coordination and supervision as in the intervention group) in addition to the usual care. FCHVs are the grass root level community health workers in Nepal who play important roles in the implementation of community-based programs including, screening for high-risk cases, referring people to local health facilities, and maintaining a record of health activities [21]. The program comparison of the intervention versus the control is summarized in Table 2.

\section{Outcome measures}

We will examine both clinical outcomes and implementation outcomes as we hypothesize that the nurse-led continuum of care program will improve both health and the implementation of priority interventions in diabetes. The outcome measure will pertain to the individual participant level. The primary outcome for the diabetes group is $\mathrm{HbA} 1 \mathrm{c}$ level at 12 months from the intervention. The $\mathrm{HbA} 1 \mathrm{c}$ level is indicative of the average level of blood glucose over the past 2 to 3 months [22]. The primary outcome for the prediabetes group is the incidence of diabetes at 12 months from the intervention.

The secondary outcomes are blood pressure, body mass index (BMI), and lipid levels. Also, the secondary outcomes include a series of implementation outcomes in the RE-AIM implementation framework (Reach, effectiveness, adoption, implementation, and maintenance) [23-25]. We provided details on those measures below (see Table 1 for a summary).

Clinical outcomes. The clinicians from Dhulikhel hospital outreach centers not otherwise associated with the project will measure $\mathrm{HbA} 1 \mathrm{c}$, lipid profiles, BMI and blood pressure when the participants visit the diabetic clinic at baseline, 6 and 12 months.

The HbA1c will be measured using Boronate affinity chromatography (Axis-Shield, Norway) [26]; LDL and HDL using the elimination method (Dialab, Austria) [27]; triglyceride using GPO-PAP (Dialab, Austria) [28]; and total cholesterol using CHOD-PAP (Dialab, Austria) [29]. For each type of assay, the laboratory has quality control (QC) materials (using commercially available assayed and unassayed control material) from Bio-Rad Laboratories, USA. Each QC is run at least in duplicate. External QC is arranged by internationally recognized reference laboratories that distribute batches of samples of various concentrations for each assay. The laboratory performs 
the External Quality Assurance Scheme from an unknown assayed sample from the Department of Clinical Biochemistry CMC, Vellore, India for 23 routine parameters, 5 immunological parameters, and HbA1c. Additionally, $5 \%$ of the blood samples will be obtained in duplicates and sent for testing all parameters, blinded to the laboratory personnel.

The mean of three measurements of systolic and diastolic blood pressure, using a Microlife automatic blood pressure measuring device, will be adopted for analysis $(\mathrm{mmHg})$. Hypertension is defined as systolic blood pressure $\geq 140 \mathrm{~mm} \mathrm{Hg}$ or, diastolic blood pressure $\geq 90 \mathrm{~mm} \mathrm{Hg}$; or taking antihypertensive medication [30]. Weight will be measured and recorded to the nearest $0.1 \mathrm{~kg}$, without shoes, and with minimum clothing, using an Omron Model HBF-400 scale. Height will be measured without shoes using a standard tape measure with participants standing against a wall for measurement and recorded to the nearest $0.1 \mathrm{~cm}$. Body Mass Index (BMI) will be calculated as weight in kilograms divided by height in meters squared. Overweight is defined as a BMI of $25 \mathrm{~kg} / \mathrm{m}^{2}$ or higher and obesity is defined as BMI of $30 \mathrm{~kg} / \mathrm{m}^{2}$ or higher based on international cut-points [31].

Implementation outcomes. RE-AIM: (1) Reach will be measured by the number of people participating in the program divided by the number of people eligible to be recruited into the program; (2) Effectiveness will be represented by the clinical outcomes; (3) Adoption at the patient level will be measured by the proportion of people adherent to the clinical advice in lifestyle and self-care. These will be measured by the self-reported Summary of Diabetes Self-Care Activities (SDSCA) scale [32] at baseline, 6 months and 12 months. The SDSCA measure is a brief self-report questionnaire that includes items assessing general diet, specific diet, exercise, blood-glucose testing, foot care, and smoking. In addition, the Diabetes Treatment Satisfaction Questionnaire (DTSQ) [33] will be administered at baseline, 6 and 12 months as well. DTSQ is the most commonly used patient-reported outcome in diabetes trials, which reflects the patient's perception of the treatment. Adoption at the clinic level will be measured by the proportion of health clinics successfully recruited into the program over clinics eligible and approached to participate in the program; (4) Implementation will examine fidelity and quality of the program execution per protocol. To check program fidelity, we will select and train standardized patient (SP) from the program participants with stable conditions. Those SPs will serve as the "secret agents" and the sentry to assess program fidelity with a quality checklist through their routine encounters to the clinicians at the DH diabetes clinic and the nurses. The clinicians will be blinded to the status of the SPs. The development of SP and checklist will follow the protocol we have developed in a separate study [34]. (5) Maintenance will not be assessed for the purpose of this study.

The hospital and program administrative system will collect a range of other information including detailed program costs, health service utilization, and incidence of complications and comorbidities. All data will be entered and securely stored in EHR, a secured online data capturing, and management system developed for the study at the $\mathrm{DH}$.

\section{Sample size}

In this proposed community-based intervention, the changes in the HbA1c levels (continuous outcome) was considered for calculating the sample size for the individuals with diabetes and the incidence of diabetes (binary outcome) was considered for calculating the sample size for the individuals with prediabetes respectively. For the individuals with diabetes, we aim to detect a clinically significant reduction in $\mathrm{HbA} 1 \mathrm{c}$ levels from $7.6 \%$ to $6.5 \%$ $(S D=1.5 \%$ ) (based on our assumption, recommended effective diabetic management level, and results of previous studies) among the individuals with diabetes during the 12 months period $[35,36]$ with medium effect size [37]. We 
expect a reduction of $\mathrm{HbA} 1 \mathrm{c}$ to $7.0 \%$ in the control group, an optimal target level defined by American Diabetes Association (ADA) [38]. Setting the statistical significance at the 0.05 level, seeking $90 \%$ power, an intraclass correlation coefficient (ICC) of 0.01 based on a previous study conducted in Nepal [39], an average cluster size of 50 (individuals with diabetes) based on the preliminary data from awareness and screening campaign, design effect for clustering of 1.49 , and design effect for unequal cluster size of 1.52 (coefficient of variation of cluster size 0.25), 91 people with diabetes per arm (182 total) will be required for the study. For the incidence of diabetes among people with prediabetes (dichotomous outcome), we considered the incidence of $10 \%$ and $2 \%$ respectively for the two groups; stated as a range in the earlier study [40]. Based on the above considerations, 471 people with prediabetes per arm (942 total) will be required. We expect a drop-out rate of $10 \%$ from baseline to 12 months follow up; hence, we will need to recruit a total of 200 diabetes and 1036 prediabetes participants (total sample size $=1236$ participants). The sample size for our cluster RCT was calculated using the equation below. Detail of the sample size estimation is available in another study conducted by Ribeiro et al. 2018 [41].

$\mathrm{SS}_{\text {cluster RCT }}=\mathrm{SS}_{\text {standard RCT }} \times \mathrm{DE}_{\text {cluster }} \times \mathrm{DE}_{\text {unequal }}$

where,

$\mathrm{SS}_{\text {cluster RCT }}=$ total sample size for cluster RCT;

$\mathrm{SS}_{\text {standard RCT }}=$ total sample size for standard RCT (equal to $\left(Z_{1-\alpha / 2}+Z_{1-\beta}\right)^{2} 2 \sigma^{2} / \Delta^{2}$ ) where Zx is the $x^{\prime}$ th percentage point of the standard normal distribution, $\Delta$ is the clinically important difference in treatment means and $\sigma^{2}$ is the variance in the outcome)

$D E_{\text {cluster }}=$ design effect for clustering (equal to $1+I C C^{\star}(m-1)$ where $m$ is the number per site)

$D E_{\text {unequal }}=$ design effect for unequal cluster size (equal to $\left.1+\left[\left(1+c v^{2}\right) \times m-1\right] \star I C C\right)$ where cv is the coefficient of variation of cluster size and $\mathrm{m}$ is the mean cluster size)

\section{Statistical analysis}

Data will be analyzed at the individual (patient) level. Statistical analyses will be performed using the intention-totreat (ITT) approach in the originally assigned groups. Demographic and baseline characteristics for the intervention and control group will be presented in the form of mean (standard deviation [SD]) or 95\% confidence intervals (Cls) for continuous variables and counts (percentages) for categorical variables. ITT analysis will be performed on the final data collected at 12 months.

Generalized estimating equations (GEE) with clustering by site, an exchangeable correlation matrix, and robust variances will be used to assess program effect adjusting for the potential baseline covariates. The model will take account of clustering and will be used to look at the difference between groups for HbA1c level at 6 and 12 months respectively. Multiple imputation will be used to account for the missing values assuming they are missing at random. To test for the secondary sensitivity analysis, data will be analyzed without multiple imputation and without baseline covariate adjustment. The difference in the incidence of diabetes between the groups among the individuals with prediabetes will be tested using a GEE. Statistical significance will be assessed at the $5 \%$ level and all analyses will be 2-sided. All data analyses will be performed using Stata 15 (StataCorp, Texas, USA) statistical software program. 
As we expect the program effect may differ between several subgroups of the program participants, we plan to do secondary analyses of the following three subgroups for the primary $(\mathrm{HbA} 1 \mathrm{C})$ and secondary outcomes (incidence of diabetes): (i) the subgroups with poor initial glycemic level ( $\mathrm{HbA} 1 \mathrm{c} \geq 5.7 \%)$ (people with poorer glycemic level at baseline may have a stronger desire to change their behavior); (ii) the subgroups divided by gender (in Nepal, women tend to be more adherent to clinician guidance); (iii) the subgroups of different social economic classes (people in higher socioeconomic class may be more likely to adhere to lifestyle changes); and (iv) government vs. non-government health centers. The secondary analyses will compare the HbA1c level and incidence of diabetes among people with prediabetes among these subgroups at the end of the 12-month intervention using the generalized linear mixed models with a random cluster effect and adjusting for potential confounders.

Descriptive statistics (i.e. counts, means and proportions) will be calculated to evaluate the reach, effectiveness, adoption, and implementation of the program.

Economic Analysis: Cost-effectiveness analysis will also be conducted with a Markov simulation model to be developed. The Markov model has been widely used to describe the development of the chronic noncommunicable diseases [42]. All direct costs (e.g., screening cost, cost of training of nurses, and cost of EHR system development) and indirect costs will be collected. All costs will be reported in 2020 US dollars using the exchange rate of 1 USD $=111.7$ NPR. We will solve the model numerically over a short and long timeframe (i.e., 10 years and 20 years), and calculate the incidence of diabetes, costs and quality-adjusted-life-years (QALYs) under the control case or NUCOD. Incremental QALY and costs of NUCOD relative to the control case will be calculated with an annual discount rate of 3\%. We will use WHO-CHOICE (Choosing Interventions that are Cost-Effective) thresholds for cost-effectiveness: an intervention is defined as "cost-effective" if it produces a healthy life year for less than three times gross domestic product (GDP) per capita, and as "very cost-effective" if it produces a healthy life year for less than the GDP per capita $[43,44]$.

\section{Data monitoring and quality assurance}

The trial will receive overall supervision by a Trial Steering Committee (TSC) who will conduct quarterly on-site monitoring of health clinics (with repeat visits to sites where performance is a concern), monitor trial progress, and will review the quality and completeness of data. TSC will include an independent chairperson not involved directly with the study, one clinical and one methodological expert who are also not involved with the study, and a principal investigator of the study. Our trained diabetes nurses will be responsible for all aspects of local organization such as identifying and recruiting participants, and RAs will be responsible for obtaining informed consent. A formal data monitoring committee (DMC) was not considered for the conduct of this study as this will be a low-risk intervention; however, the trial will be reviewed by a national (every 6-month) and institutional (every 2-month) Ethics Committee Review Board members throughout the trial period. Stakeholder and Public Involvement Group (SPIG) was also not formed as the study will be mainly led by the trained diabetes nurses.

\section{Discussion}

To our knowledge, this study is the first to include a sizable number of diabetes and prediabetes participants in a community-based setting, and a continuum of care program run by diabetes nurses. The priority health problems of the target population, potential solution of the problem, recruitment strategies, and intervention delivery formats were early on discussed by the study-specific collaborative partners in $\mathrm{DH}$ and community, which will lead to the success of the program.

Page $11 / 22$ 
The results of this study may provide much-needed evidence to the policy-makers on the clinical effectiveness, cost-effectiveness and implementation effectiveness of nurse-led continuum of care for people with diabetes and prediabetes in resource-limited settings. Many low-income countries now face a double burden of chronic diseases and infectious diseases. But large trials have been rarely conducted in those settings in low-income countries. This proposal has several unique features. First: Although nurse-led diabetes prevention and management programs have been tested earlier in different settings, this is unique because it addresses the full continuum of care from the level of awareness and detection to management and follow-up all led by nurses. Second: The study focuses not only on clinical outcomes but also on implementation outcomes. The nurse-led continuum of care may theoretically improve health among people with diabetes but also improvement adoption of DCT-3 recommended and evidence-based individual intervention elements. We will also use some innovative strategies to assess program implementation fidelity and quality such as the use of standardized patients. Third: It builds a culturally tailored and locally contextual training program for nurses utilizing the existing training programs of the International Diabetes Federation and the American Diabetes Association. Fourth: It utilizes a unique setting of sub-urban tertiary level community-based health institution that has an extensive network of health centers in rural areas as well. Hence, the experience we gain from this project will be easily translated into larger health care systems. Fifth: We have designed the trial, taking implementation, feasibility, and sustainability into full consideration. For instance, we did not offer free care to all program participants in the trial as that will not be sustainable once the program stops.

Although this trial may hold promise for better clinical and implementation outcomes, it is not without potential limitations. We will have a large sample size, but the setting of the study may not represent the entire nation, thus limiting the generalizability of our findings. However, our setting will cover both public and private health sectors and may serve as a proxy for the Nepalese population.

\section{Trial Status}

The protocol is the first version as we have not changed any information after registering into the ClinicalTrials.gov registry on 18 October 2019. We have not yet started the recruitment of our sample. The approximate date when recruitment will be completed will be the end of December 2020.

\section{List Of Abbreviations}

ADA: American Diabetes Association

BMI: Body Mass Index

DCP-3: Disease Control Priority-3

DH: Dhulikhel Hospital

DPP: Diabetes Prevention Program

DRT: Diabetes Risk Test

EHR: Electronic Health Record

FCHV: Female Community Health Volunteer 
HDL: High-Density Lipoprotein

ICC: Intraclass Correlation Coefficient

IDRS: Indian Diabetes Risk Score

ITT: Intention to Treat

LDL: Low-Density Lipoprotein

MET: Metabolic Equivalent of Task

NPR: Nepalese Rupee

QALY: Quality-Adjusted Life Years

QC: Quality Control

USD: United States Dollar

\section{Declarations}

Ethics approval and consent to participate: This study will involve field procedures in Nepal and data analyses in Dhulikhel, Nepal and Guangzhou, China. This protocol received central ethical approval from the Nepal Health Research Council (NHRC\# 464/2019) and local ethical approval from the Kathmandu University School of Medical Sciences (\#216/19) in Nepal, and from the School of Public Health at Sun Yat-sen University in Guangdong, China (SYSU\# 2018-037). However, we will not begin recruiting at other centers in the trial until the local approval from each center has been obtained. All participants will provide written informed consent before participating in the study.

Consent for publication: All participants will provide written informed consent before participating in the study, which will include consent to publish anonymous results from individual participants.

Availability of data and materials: The proposed project is a collaborative effort between investigators at the SYSU and KU. The aforementioned institutions will jointly share ownership of the data. Study investigators at the SYSU and $\mathrm{KU}$ will have access to the data. Data can be obtained from the corresponding author upon reasonable request.

Competing interests: The authors declare that they have no competing interests.

Funding: This study will be conducted under the umbrella of big implementation project and will be supported by the World Diabetes Foundation (WDF17-1483) grant, China Medical Board (CMB16-260) grant, and Dhulikhel Hospital Kathmandu University. However, the funding organizations will have no role in the design of the study, data collection, data analysis, data interpretation, or writing of the report.

Author's contributions: All authors contributed to the preparation of this manuscript (BMK, RD, AS, LJ, AS, ZL, PS, DX). DX and BKM led the conception and design of the study. DX, RD and AS prepared the first draft of the protocol. RD participated in revising and finalizing the draft of the manuscript by critically revising the important 
intellectual content. BMK, JL, PS and AS provided detailed comments on the draft for the revision. All authors read and approved the final version of the manuscript.

Acknowledgments: We gratefully acknowledge diabetes study team members at Dhulikhel Hospital for their assistance with study coordination.

\section{Protocol amendments}

Any change to a protocol requiring approval will be notified to the national and ethical review committees and will be updated into the ClinicalTrials.gov registry.

\section{Dissemination policy}

Upon completion of the study, the results of the study will be presented to the healthcare professionals at Dhulikhel Hospital, Ministry of Health and Population (MoHP), Nepal, at national and international conferences, and submitted to international peer-reviewed journals.

\section{References}

1. Gyawali B, Sharma R, Neupane D, Mishra SR, van Teijlingen E, Kallestrup P. Prevalence of type 2 diabetes in Nepal: a systematic review and meta-analysis from 2000 to 2014. Glob Health Action. 2015;8:29088.

2. Institute for Health Metrics and Evaluation (IHME). Globle Burden of Disease Profile 2018. http://www.healthdata.org/sites/default/files/files/country_profiles/GBD/ihme_gbd_country_report_nepal.pdf. Accessed 1 June 2018.

3. Aubert RE, Herman WH, Waters J, Moore W, Sutton D, Peterson BL, et al. Nurse case management to improve glycemic control in diabetic patients in a health maintenance organization. A randomized, controlled trial. Ann Intern Med. 1998;129(8):605-12.

4. Li D, Elliott T, Klein G, Ur E, Tang TS. Diabetes Nurse Case Management in a Canadian Tertiary Care Setting: Results of a Randomized Controlled Trial. Can J Diabetes. 2017;41(3):297-304.

5. Conway A, O'Donnell C, Yates P. The Effectiveness of the Nurse Care Coordinator Role on Patient-Reported and Health Service Outcomes: A Systematic Review. Eval Health Prof. 2019;42(3):263-96.

6. Jutterstrom L, Hornsten A, Sandstrom H, Stenlund H, Isaksson U. Nurse-led patient-centered self-management support improves $\mathrm{HbA} 1 \mathrm{C}$ in patients with type 2 diabetes-A randomized study. Patient Educ Couns. 2016;99(11):1821-9.

7. Bilous M TI, Winship S, Ellis J, Evans T, Nag S, Jones S, Bilous R. A nurse-led cardiovascular management programme significantly reduces risk in people with Type 2 diabetes and reduced estimated glomerular filtration rate. Diabetic Medicine. 2011;28(195).

8. Campbell MK, Elbourne DR, Altman DG, group C. CONSORT statement: extension to cluster randomised trials. BMJ. 2004;328(7441):702-8.

9. Pinnock H, Barwick M, Carpenter CR, Eldridge S, Grandes G, Griffiths CJ, et al. Standards for Reporting Implementation Studies (StaRI) Statement. BMJ. 2017;356:i6795.

10. Wikipedia. Administrative divisions of Nepal. https://en.wikipedia.org/wiki/Administrative_divisions_of_Nepal. Accessed 12 June 2019. 
11. Dudeja P, Singh G, Gadekar T, Mukherji S. Performance of Indian Diabetes Risk Score (IDRS) as screening tool for diabetes in an urban slum. Med J Armed Forces India. 2017;73(2):123-8.

12. Basina M. Understanding a Type 2 Diabetes Diagnosis. https://www.healthline.com/health/type-2diabetes/diagnosis. Accessed 12 Jun 2019.

13. Kermani SK, Khatony A, Jalali R, Rezaei M, Abdi A. Accuracy and Precision of Measured Blood Sugar Values by Three Glucometers Compared to the Standard Technique. J Clin Diagn Res. 2017;11(4):0C05-0C8.

14. Gyawali B, Hansen MRH, Povlsen MB, Neupane D, Andersen PK, McLachlan CS, et al. Correction: Awareness, prevalence, treatment, and control of type 2 diabetes in a semi-urban area of Nepal: Findings from a crosssectional study conducted as a part of COBIN-D trial. PLoS One. 2018;13(12):e0209046.

15. CommCare. The worlds most powerful data collection platform. https://www.dimagi.com/commcare/. Accessed 16 Oct 2019.

16. Bull FC, Maslin TS, Armstrong T. Global physical activity questionnaire (GPAQ): nine country reliability and validity study. J Phys Act Health. 2009;6(6):790-804.

17. Rifas-Shiman SL, Willett WC, Lobb R, Kotch J, Dart C, Gillman MW. PrimeScreen, a brief dietary screening tool: reproducibility and comparability with both a longer food frequency questionnaire and biomarkers. Public Health Nutr. 2001;4(2):249-54.

18. Association AD. Are you at risk for type 2 diabetes? http://main.diabetes.org/dorg/PDFs/risk-test-paperversion.pdf. Accessed 20 Jul 2018.

19. Goldstein MG, Whitlock EP, DePue J, Planning Committee of the Addressing Multiple Behavioral Risk Factors in Primary Care P. Multiple behavioral risk factor interventions in primary care. Summary of research evidence. Am J Prev Med. 2004;27(2 Suppl):61-79.

20. Ackermann RT, Marrero DG. Adapting the Diabetes Prevention Program lifestyle intervention for delivery in the community: the YMCA model. Diabetes Educ. 2007;33(1):69, 74-5, 77-8.

21. Khatri RB, Mishra SR, Khanal V. Female Community Health Volunteers in Community-Based Health Programs of Nepal: Future Perspective. Front Public Health. 2017;5:181.

22. Sherwani SI, Khan HA, Ekhzaimy A, Masood A, Sakharkar MK. Significance of HbA1c Test in Diagnosis and Prognosis of Diabetic Patients. Biomark Insights. 2016;11:95-104.

23. Harden SM, Smith ML, Ory MG, Smith-Ray RL, Estabrooks PA, Glasgow RE. RE-AIM in Clinical, Community, and Corporate Settings: Perspectives, Strategies, and Recommendations to Enhance Public Health Impact. Front Public Health. 2018;6:71.

24. Glasgow RE EP. Peer Reviewed: Pragmatic Applications of RE-AIM for Health Care Initiatives in Community and Clinical Settings. Prev Chronic Dis. 2018;15(E02).

25. Gaglio B, Shoup JA, Glasgow RE. The RE-AIM framework: a systematic review of use over time. Am J Public Health. 2013;103(6):e38-46.

26. SKUP. Afinion ${ }^{\text {TM }}$ system for HbA1c. A system for measurement of B-haemoglobin A1c manufactured by AxisShield PoC AS, Norway. file:///Users/rubeedev/Downloads/skup.pdf. Accessed 1 Apr 2019.

27. DIALAB. Cholesterol HDL, Direct immunoinhibition 2 Reagent. Diagnostic reagent for quantitative in vitro determination of high density lipoprotein cholesterol (HDL-C) in human serum or plasma or photometric systems. DIALAB Produktion und Vertrieb von chemisch - technischen Produkten und Laborinstrumenten Gesellschaft m.b.H. Austria 2018. http://www.diameb.ua/manuals/eng/F03100.pdf. Accessed 20 Apr 2020. 
28. DIALAB. Triglycerides GPO-PAP with ATCS Single Reagent. Diagnostic Reagent for quantitative in vitro determination of Triglycerides in human serum or plasma on photometric systems. DIALAB Produktion und Vertrieb von chemisch - technischen Produkten und Laborinstrumenten Gesellschaft m.B.H.. Austria 2018. http://www.diameb.ua/manuals/eng/D00389.pdf. Accessed 20 Apr 2020.

29. DIALAB. Cholesterol CHOD-PAP with ATCS Single Reagent. Diagnostic Reagent for Quantitative in Vitro Determination of Cholesterol in Serum or Plasma on Photometric Systems. DIALAB Produktion und Vertrieb von chemisch - technischen Produkten und Laborinstrumenten Gesellschaft m.b.H. Austria 2016. http://www.diameb.ua/manuals/eng/D98118.pdf. Accessed 20 Apr 2020.

30. Chobanian AV BG, Black HR, Cushman WC, Green LA, Izzo Jr JL, Jones DW, Materson BJ, Oparil S, Wright Jr JT. The seventh report of the joint national committee on prevention, detection, evaluation, and treatment of high blood pressure. The JNC 7 Report. JAMA. 2003;289(19):2560-71.

31. WHO Expert Consultation. Appropriate body-mass index for Asian populations and its implications for policy and intervention strategies. Lancet. 2004;363(9403):157-63.

32. Toobert DJ, Hampson SE, Glasgow RE. The summary of diabetes self-care activities measure: results from 7 studies and a revised scale. Diabetes Care. 2000;23(7):943-50.

33. C B. The diabetes treatment satisfaction questionnaire: DTSQ. In C. Bradley (Ed.) HoPaDagtpmidrap, editor1994.

34. Xu DR, Hu M, He W, Liao J, Cai Y, Sylvia S, et al. Assessing the quality of primary healthcare in seven Chinese provinces with unannounced standardised patients: protocol of a cross-sectional survey. BMJ Open. 2019;9(2):e023997.

35. Sapkota RP, Upadhyaya T, Gurung G, Parker M, Raman R, Pardhan S. Need to improve awareness and treatment compliance in high-risk patients for diabetic complications in Nepal. BMJ Open Diabetes Res Care. 2018;6(1):e000525.

36. Rajbhandari PMS GP, Mahato RV, Chaudhary D. A Cross-Sectional Prospective Study of Glycated Hemo-globin (Hba1c) and Fasting Blood Glucose (Fbg) Level In Both Dia-betic and Non-Diabetic Patients in Context to Nepalese General Population. MJ Diab. 2017;2(2).

37. Cohen J. Statistical power for the Behavioural Sciences. Hampson SE, editor. Hillsdale NY: Lawrence Erlbaum. 1988.

38. American Diabetes Association (ADA). Glycemic targets: Standards of Medical Care in Diabetes-2019. Diabetes Care. https://care.diabetesjournals.org/content/42/Supplement_1/S61. Accessed 20 Apr 2020.

39. Gyawali B, Neupane D, Vaidya A, Sandbaek A, Kallestrup P. Community-based intervention for management of diabetes in Nepal (COBIN-D trial): study protocol for a cluster-randomized controlled trial. Trials. 2018;19(1):579.

40. Tabák AG HC, Rathmann W, Brunner EJ, Kivimäki M. Prediabetes: a high-risk state for developing diabetes. Lancet. 2012;379:2279.

41. Ribeiro DC, Milosavljevic S, Abbott JH. Sample size estimation for cluster randomized controlled trials. Musculoskelet Sci Pract. 2018;34:108-11.

42. Briggs AD, Wolstenholme J, Blakely T, Scarborough P. Choosing an epidemiological model structure for the economic evaluation of non-communicable disease public health interventions. Popul Health Metr.

2016;14:17. 
43. Baltussen RM AT, Tan-Torres Edejer T, Hutubessy RCW, Acharya A, Evans DB, Murray CJL. World Health Organization. Making choices in health: WHO guide to cost-effectiveness analysis. 2003.

https://www.who.int/choice/publications/p_2003_generalised_cea.pdf. Accessed 20 Apr 2020.

44. Hutubessy RC BR, Torres-Edejer TT, Evans DB. WHO-CHOICE: Choosing interventions that are cost-effective. Health Systems Performance Assessment: Debates, Methods and Empiricism. Geneva, Switzerland. WHO Editions; 2003:823-35.

\section{Tables}

Table 1: Measures of outcome variables

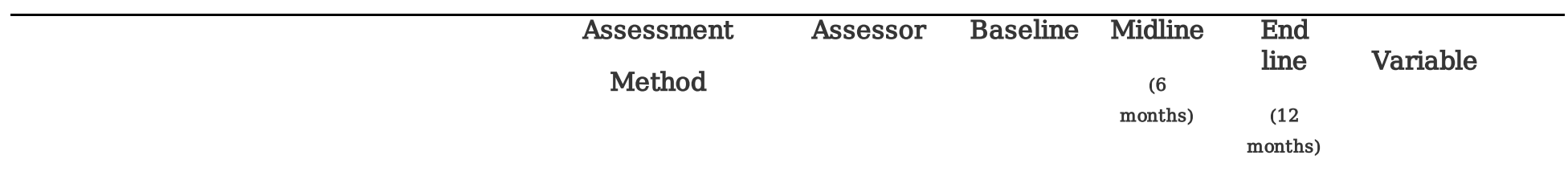

\section{Clinical outcomes}

\section{Primary outcome}

Glycemic control (HbA1c) ${ }^{1}$ for

Blood sample

Biochemist

$\mathrm{x}$

$\mathrm{x}$

$\mathrm{x}$

Continuous

diabetes

Incidence of diabetes among

EHR

RA/Nurse

$\mathrm{X}$

$\mathrm{X}$

Binary

prediabetes

\section{$\underline{\text { Secondary outcomes }}$}

Blood pressure

Lipid profile

Body mass index

Electronic monitor

Blood sample

$\mathrm{EHR}^{2}$

\section{Implementation outcomes}

Reach

Adoption

Implementation (fidelity)

Self-monitoring of glucose

Medication adherence

\begin{tabular}{|c|c|}
\hline Log Record ${ }^{3}$ & RA/Nurse \\
\hline SDSCA/ DTSQ & RA/Nurse \\
\hline Checklist & SP \\
\hline Self-reported & RA/Nurse \\
\hline Self-reported & RA/Nurse \\
\hline
\end{tabular}

$\begin{array}{ccc}\mathrm{x} & \mathrm{x} & \text { Continuous } \\ \mathrm{x} & \mathrm{x} & \text { Binary } \\ \mathrm{x} & \mathrm{x} & \text { Continuous } \\ \mathrm{x} & \mathrm{x} & \text { Binary } \\ \mathrm{x} & \mathrm{x} & \text { Binary }\end{array}$

1 The average level of blood sugar over the past 2 to 3 months

2 Calculated as weight in kilograms divided by height in meters squared

3 Number of people participating in the program divided by the number of people eligible to be recruited into the program DTSQ= Diabetes Treatment Satisfaction Questionnaire; HER= Electronic Health Record; HbA1c= HemoglobinA1 c (aka Glycated Hemoglobin); RA= Research Assistant; SP= Standardized Patient; SDSCA= Summary of Diabetes Self-Care Activities Assessment 
Table 2: Program components for intervention vs. control group

\begin{tabular}{lcc}
\hline & Intervention & Control \\
\hline Community awareness campaigns led by trained nurses & $\mathrm{x}$ & $\mathrm{x}$ \\
Screening & $\mathrm{x}$ & $\mathrm{x}$ \\
Linkage to clinical care: & $\mathrm{x}$ & \\
& $\mathrm{x}$ & \\
- coordinated through the nurses & & $\mathrm{x}$ \\
- provided by FCHVs without the involvement of the nurse & $\mathrm{x}$ & $\mathrm{x}$ \\
Improved quality of care at the hospital & $\mathrm{x}$ & \\
Nurse-led counseling & $\mathrm{x}$ & \\
Community follow-up coordinated through the nurses & $\mathrm{x}$ & \\
\hline
\end{tabular}

FCHVs $=$ Female Community Health Volunteers

\section{Figures}




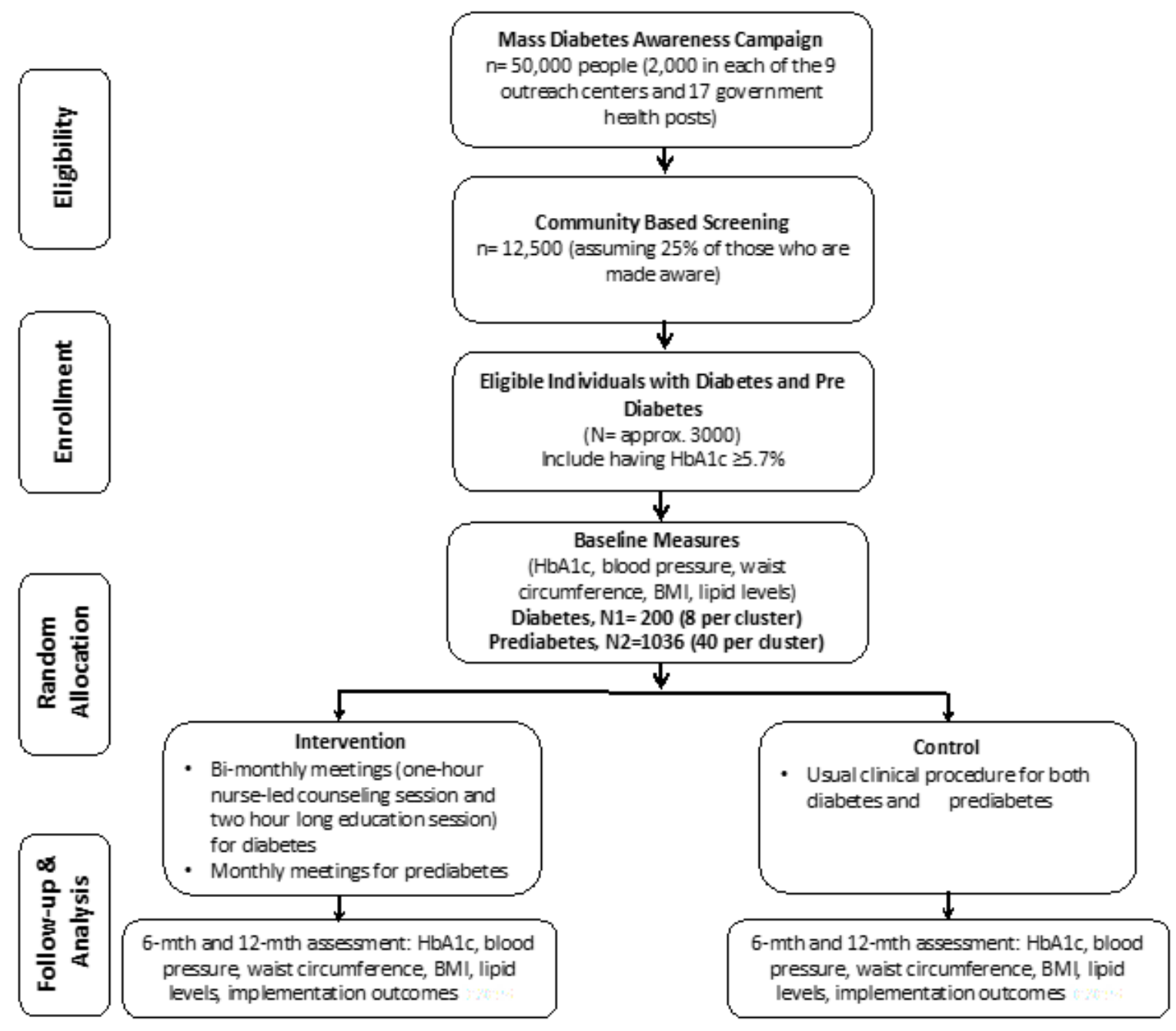

Figure 1

Summary of trial design for NUCOD program 


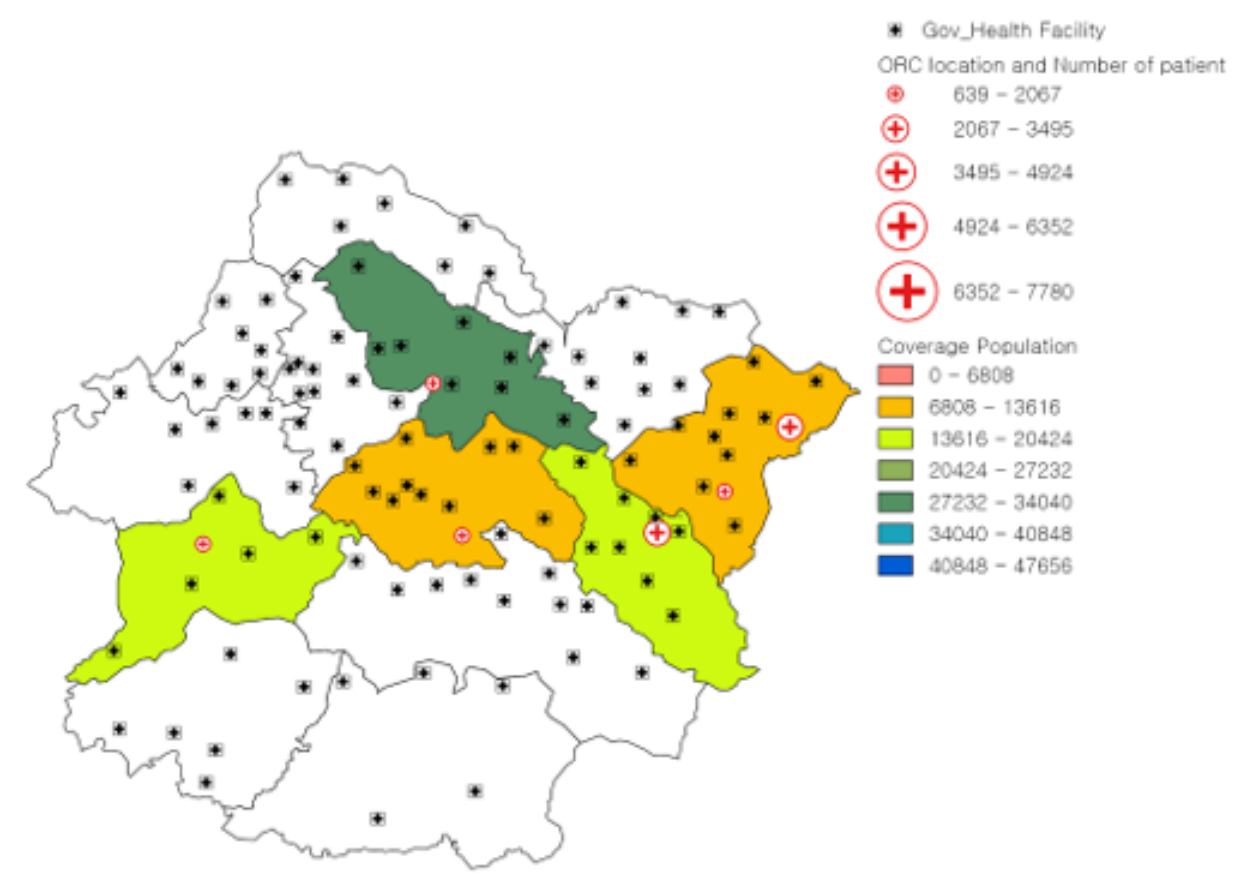

Figure 2

Map of Kavre District with Outreach Centers of Dhulikhel Hospital and Government Facilities. ORC= Outreach Center

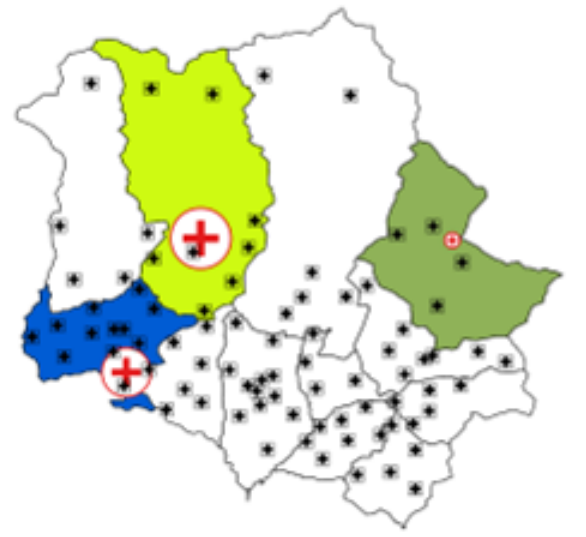

$$
\begin{aligned}
& \text { * Gow_Health Facility } \\
& \text { OAC location and Number of patient } \\
& \text { (†) } 639-2067 \\
& \text { † } 2067-3495 \\
& \text { (†) } 3495-4924 \\
& \text { (†) } 4924-6352 \\
& \text { (4) } 6352-7780 \\
& \text { Coverage Population } \\
& \square 0-6808 \\
& \square 6808-13616 \\
& \square 13616-20424 \\
& \square 20424-27232 \\
& \square 27232-34040 \\
& 34040-40848 \\
& 40848-47656
\end{aligned}
$$

\section{Figure 3}


Training of nurses

Develop a tailored training protocol for the nurses

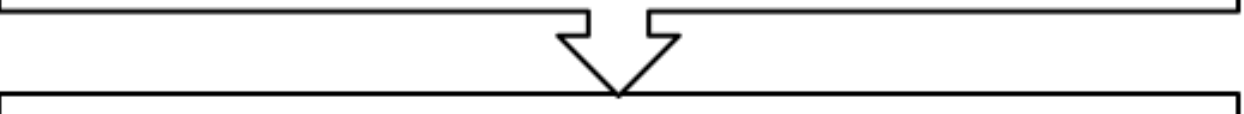

Community awareness campaigns

Conduct mass-campaigns on diabetes and prediabetes

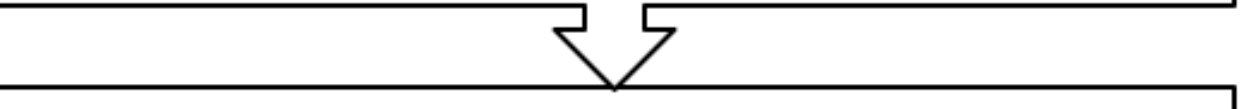

Screening programs

Organize screening programs for individuals with diabetes and prediabetes in the community

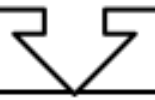

Linkage to clinical Care

Nurses will refer (or arrange a visit) positively screened individuals with diabetes and prediabetes (pre-existing as well as new) to attend a diabetes clinic at $\mathrm{DH}$

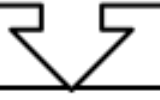

Community follow-up counselling and support for the individuals with diabetes and prediabetes

Nurses will organize individuals in a group of 10 and arrange meetings (about two-hour long) bi-monthly for individuals with diabetes and once a month for individuals with prediabetes. In this group counseling, individuals will be facilitated using 5 As model (Assess, Advise, Agree, Assist, Arrange) to adopt the healthy lifestyle.

Figure 4

Intervention components of the NUCOD program. DH= Dhulikhel Hospital 


\begin{tabular}{|c|c|c|c|c|c|c|c|}
\hline \multirow[b]{3}{*}{ TIMEPOINT } & \multicolumn{7}{|c|}{ STUDY PERIOD } \\
\hline & \multirow{2}{*}{$\begin{array}{c}\text { Enrolment } \\
-M_{3}\end{array}$} & \multirow{2}{*}{$\begin{array}{c}\text { Allocation } \\
0\end{array}$} & \multicolumn{4}{|c|}{ Post-allocation } & \multirow{2}{*}{$\begin{array}{c}\text { Close-out } \\
M_{15}\end{array}$} \\
\hline & & & $M_{3}$ & M6 & Mg & $M n$ & \\
\hline \multicolumn{8}{|l|}{ ENROLMENT: } \\
\hline $\begin{array}{r}\text { Community awareness and } \\
\text { screening }\end{array}$ & $\mathrm{X}$ & & & & & & \\
\hline \multirow{2}{*}{$\begin{array}{r}\text { Informed consent } \\
\text { Allocation }\end{array}$} & $\mathrm{X}$ & & & & & & \\
\hline & & $\mathrm{X}$ & & & & & \\
\hline \multicolumn{8}{|l|}{ INTERVENTIONS: } \\
\hline \multirow[b]{3}{*}{ Hospital quality improvement } & $\mathrm{X}$ & & & & & & \\
\hline & $\mathrm{X}$ & & & & & & \\
\hline & $\mathrm{X}$ & & & & & & \\
\hline \multicolumn{8}{|l|}{ Community follow-up } \\
\hline \multicolumn{8}{|l|}{ ASSESSMENTS: } \\
\hline IDRS & $\mathrm{X}$ & & & & & & \\
\hline HbAlc & $\mathrm{X}$ & & & $\mathrm{X}$ & & $\mathrm{X}$ & \\
\hline Blood pressure & $\mathrm{X}$ & & & $\mathrm{X}$ & & $\mathrm{X}$ & \\
\hline Lipid profile & $\mathrm{X}$ & & & $\mathrm{X}$ & & $\mathrm{X}$ & \\
\hline Weight/height & $\mathrm{X}$ & & & $\mathrm{X}$ & & $\mathrm{X}$ & \\
\hline SDSCA (lifestyle/self-care) & $\mathrm{X}$ & & & $\mathrm{X}$ & & $\mathrm{X}$ & \\
\hline Prime screen (diet assessment) & $\mathrm{X}$ & & & $\mathrm{X}$ & & $\mathrm{X}$ & \\
\hline DTSQ (treatment satisfaction) & $\mathrm{X}$ & & & $\mathrm{X}$ & & $\mathrm{X}$ & \\
\hline SP (program fidelity/quality) & & & & & & & \\
\hline
\end{tabular}

M=Month; IDRS=Indian Diabetes Risk Score; HbA1c= Glycated Hemoglobin; SDSCA=Summary of Diabetes Selfcare Activities; DTSQ=Diabetes Treatment Satisfaction Questionnaire; SP=Standardized patients

Figure 5

Schedule of enrolment, interventions, and assessments

\section{Supplementary Files}

This is a list of supplementary files associated with this preprint. Click to download.

- StaRIChecklistforNUCODTrial.pdf

- SPIRITchecklistforNUCOD.pdf 